\title{
Choosing the Most Suitable Classifier for Supporting Assistive Technology Adoption in People with Parkinson's Disease: A Fuzzy Multi-criteria Approach
}

Miguel Ortíz-Barrios, Ian Cleland, Mark Donnelly, Jonathan Greer, Antonella Petrillo, Zaury Fernández-Mendoza and Natalia Jaramillo-Rueda

\begin{abstract}
Parkinson's disease (PD) is the second most common neurodegenerative disorder which requires a long-term, interdisciplinary disease management. While there remains no cure for Parkinson's disease, treatments are available to help reduce the main symptoms and maintain quality of life for as long as possible. Owing to the global burden faced by chronic conditions such as PD, Assistive technologies (AT's) are becoming an increasingly common prescribed form of treatment. Low adoption is hampering the potential of digital technologies within health and social care. It is then necessary to employ classification algorithms have been developed for differentiating adopters and non-adopters of these technologies; thereby, potential negative effects on people with PD and cost overruns can be further minimized. This paper bridges this gap by extending the Multi-criteria decision-making approach adopted in technology adoption modeling for people with dementia. First, the fuzzy Analytic Hierarchy Process (FAHP) is applied to estimate the initial relative weights of criteria and sub-criteria. Then, the Decisionmaking Trial and Evaluation Laboratory (DEMATEL) is used for evaluating the interrelations and feedback among criteria and sub-criteria. The Technique for Order of Preferences by Similarity to Ideal Solution (TOPSIS) is finally implemented to rank three classifiers (Lazy IBk - knearest neighbors, Naïve bayes, and J48 decision tree) according to their ability to model technology adoption. A real case study considering is presented to validate the proposed approach.
\end{abstract}

Keywords: Parkinson's disease (PD) · Technology adoption · Fuzzy Analytic

Hierarchy Process (FAHP) · Decision Making Trial and Evaluation Laboratory 